\title{
ReMed: une assistance au bon moment
}

\author{
Les médecins veulent aider. Ce faisant, ils peuvent commettre des erreurs qui les affec- \\ teront gravement eux aussi, et pas seulement leurs patients. ReMed offre son assis- \\ tance dans ce genre de situation. Ce réseau de soutien propose un accompagnement \\ pour surmonter la crise et analyser les événements qui se sont produits.
}

Jürg Beutler

Communication de la FMH

\section{Expériences}

Un jeune médecin-assistant est confronté à un décès qui lui pèse. Des années plus tard, le souvenir de cette nuit l'oppresse encore.

J'étais de garde cette nuit-là, et j'étais seul responsable de 150 patients, le chef de clinique était déjà parti. Tout à coup, je reçois un appel de la garde de nuit: un patient gravement malade du cœur est couché par terre, inconscient. Je cours immédiatement dans sa chambre. Je ne peux toutefois que constater son décès. Dans mon désarroi, j'éprouve aussitôt un sentiment

\begin{abstract}
A chaque prise de contact, ReMed s'annonce dans les 72 heures; il discute avec le médecin concerné de sa situation personnelle de manière confidentielle et sans engagement, et lui présente les démarches individuelles possibles. ReMed est lié au secret médical. Avez-vous besoin d'un soutien? Ou un médecin de votre entourage?
\end{abstract}

Alors prenez contact avec ReMed:

Ligne d'assistance téléphonique 24 heures sur 24: 08000 73633, info@swiss-remed.ch, www.swissremed.ch

\footnotetext{
* cf. les initiatives qualité de la FMH

www.fmh.ch $\rightarrow$ Autres

thèmes $\rightarrow$ Qualité

$\rightarrow$ Initiatives qualité

$\rightarrow$ Gestion interne

des incidents critiques

$\rightarrow$ la deuxième victime
}

Correspondance:

Dr Michael Peltenburg

Spécialiste en médecine

générale FMH

CH-8340 Hinwil

michael.peltenburg@hin.ch de culpabilité. Ai-je oublié quelque chose? Aurais-je dû encore mieux le surveiller? Ai-je commis une faute? Naturellement, je sais bien qu'un patient aussi gravement atteint peut mourir, mais sur le moment, cette pensée ne m'est d'aucune aide.

Le lendemain, je présente les événements au rapport et je demande si j'ai pris une décision erronée. Mais mon supérieur constate seulement que ce sont des choses qui peuvent arriver. Jamais on ne me questionnera personnellement sur cet incident.

Par la suite, je me sens misérable: j'ai mauvaise conscience et j'ai peur de moi-même en tant que médecin. La nuit, je ne peux plus dormir et le jour, j'ai de la peine à me concentrer et j'ai des sueurs froides. Je manque totalement d'assurance. J'ai envie d'abandonner ma profession.

En même temps, je suis aussi furieux que l'on m'ait laissé seul alors que je débutais. Dans une telle situation, on a besoin, en tant que médecin, d'un soutien avec une direction clairvoyante de la part des supérieurs hiérarchiques. Une analyse pour voir si une faute avait été commise m'aurait déchargé, même s'il en était ressorti que j'avais mal évalué la situation. Ma position aurait été claire et j'aurais pu apprendre quelque chose.

Dans ce genre de situation, les médecins doivent avoir la possibilité d'analyser les incidents. Les services de consultation des hôpitaux fournissent naturelle- ment une aide précieuse. Mais il est aussi important d'avoir des interlocuteurs externes tels que ReMed.

\section{Interview de Daniel Herren}

En votre qualité de membre du Comité central de la FMH, vous assumez la responsabilité du domaine Données, démographie et qualité. Pourquoi est-il si important que les médecins puissent analyser ce genre d'incidents?

Mon expérience montre clairement combien une telle situation peut être lourde à porter: quand les médecins prennent des décisions erronées, il en va d'êtres humains, de destins personnels, et on ne peut pas simplement oublier, ni pendant le travail ni après. Il est donc absolument indispensable que les médecins analysent l'incident afin d'en tirer une leçon et de pouvoir à nouveau se concentrer sur leur travail. ReMed est le service de consultation à cet égard, car les interlocuteurs sont également des médecins qui connaissent ces situations suite à leurs propres expériences.

ReMed offre donc aussi un soutien aux médecins lorsqu'ils sont confrontés à une erreur de traitement. Existe-t-il d'autres plates-formes qui traitent des erreurs commises dans l'activité médicale quotidienne?

En sa qualité d'organisation professionnelle du corps médical, la FMH s'engage fortement dans les activités en faveur de la qualité. Depuis 2008, nous publions par exemple régulièrement des initiatives qualité touchant les différents domaines de l'activité médicale. En outre, la FMH est coresponsable de la fondation pour la sécurité des patients, laquelle œuvre très concrètement pour la promotion de cette sécurité. Il est prévu d'intensifier la collaboration entre ReMed et la fondation, à l'instar du projet «La deuxième victime».*

ReMed a été lancé en 2007 sous forme de projet pilote. Pouvez-vous déjà dire si l'on prévoit de le maintenir?

ReMed correspond à un besoin du corps médical et la phase pilote l'a très bien montré. Pour cette raison, nous avons décidé de l'étendre à l'ensemble de la Suisse. Des médecins de tous les cantons se sont adressés à ce réseau d'assistance déjà durant la phase pilote. Nous voulons maintenant le faire encore mieux connaitre et l'introduire sur tout le territoire national. Le coup d'envoi sera donné cet automne. 
Parler de ses expériences lors des rencontres ReMed

Mon patient est médecin. Pièges et difficultés d'une relation pas toujours facile.

9 septembre 2010, 14h30 à 18h00, Crowne Plaza, Badenerstrasse 420, 8040 Zurich

Nous serions heureux de vous y accueillir et vous remercions par avance de vous inscrire d'ici le 20 août 2010 par courriel à: ddq@fmh.ch

\section{Portrait: Fondation pour la sécurité des patients}

L'erreur est humaine et elle apparaît partout où l'on travaille. Cet adage vaut également pour les traitements prodigués aux patients. Certes, les soins de santé sont performants et de qualité élevée en Suisse. Mais la complexité croissante et les possibilités médicales accrues comportent aussi des risques. De ce fait, la sécurité des patients est devenue un thème important au cours des dernières années.

La Fondation pour la sécurité des patients, fondée en 2003, tient à fournir une contribution propre à limiter les erreurs dans la fourniture des soins. Active dans tout le pays, elle est aujourd'hui l'organisation la plus largement soutenue dans le domaine de la sécurité des patients. La Confédération et les cantons en font partie, tout comme les organisations professionnelles et les fédérations les plus importantes de la santé ainsi que l'Académie Suisse des Sciences Médicales. Le corps médical s'y engage sur plusieurs plans: la FMH, la fmCH et le Collège de médecine de premier recours sont représentés au Conseil de fondation et participent aux projets aussi bien sur le plan du contenu qu'au niveau financier.

\section{Apprendre à partir de ses erreurs}

Pour augmenter la sécurité des patients en Suisse, la Fondation encourage l'apprentissage sur la base des erreurs et de la gestion des risques cliniques. L'objectif est de réduire les erreurs dans le domaine de la santé. En sa qualité d'organisation de réseaux, la Fondation pour la sécurité des patients collabore avec de nombreux partenaires de tous les secteurs de la santé et dans de nombreux champs thématiques différents. L'accent est mis sur des projets concernant la transmission des connaissances, la recherche et la diffusion de solutions.

\section{Projets actuels}

- CIRRNET: réseau de systèmes locaux d'annonces d'erreurs, Quick Alerts (recommandations concises pour les thèmes spécifiques liés à la sécurité des patients);

- Safe Surgery et prévention des confusions entre interventions chirurgicales;

- Error and Risk Analysis: analyse systémique des erreurs dans des cours et analyses organisés sur place;

- L'auteur en tant que victime: prise en charge interne des collaborateurs après des erreurs de traitement (cours de formation et brochures);

- Communication avec les patients et les membres de leurs familles après des erreurs: cours de formation et brochures;

- Benchmarking de la sécurité des patients sur le plan intrinsèque;

- Patient empowerment: recommandations aux patients concernant leur sécurité (brochure et assistance de l'établissement);

- Drug Event Monitoring pour la sécurité de la médication et des médicaments;

- Sécurité des patients dans les soins de premier recours;

- Prévention des chutes;

- Migrants dans les pharmacies en tant que groupe à risque pour des erreurs;

- Paper of the Month, conseil, activité de formation et d'enseignement universitaire, sessions.

\section{Contact}

Fondation pour la sécurité des patients, Asylstrasse 77, 8032 Zurich, Tél. 04324376 70, info@ patientensicherheit.ch / www.patientensicherheit.ch 